\title{
Intelligent products: The grace experience
}

\author{
Paulo Leitão ${ }^{\mathrm{a}, \mathrm{b}, *}$, Nelson Rodrigues ${ }^{\mathrm{a}, \mathrm{b}}$, José Barbosa $^{\mathrm{a}}$, Claudio Turrin ${ }^{\mathrm{c}}$, Arnaldo Pagani ${ }^{\mathrm{c}}$ \\ a Polytechnic Institute of Bragança, Campus Sta Apolónia, Apartado 1134, 5301-857 Bragança, Portugal \\ ${ }^{\mathrm{b}}$ LIACC - Artificial Intelligence and Computer Science Laboratory, R. Campo Alegre 102, 4169-007 Porto, Portugal \\ c Whirlpool Europe, Cassinetta di Biandronno, Italy
}

\section{A R T I C L E I N F O}

\section{Article history:}

Received 16 November 2014

Accepted 5 May 2015

\section{Keywords:}

Intelligent products

Intelligent and reconfigurable control

Multi-agent systems

Factory automation

\begin{abstract}
A B S T R A C T
Product intelligence is a new industrial manufacturing control paradigm aligned with the context of cyber-physical systems and addressing the current requirements of flexibility, reconfigurability and responsiveness. This paradigm introduces benefits in terms of improvement of the entire product's lifecycle, and particularly the product quality and customization, aiming the customer satisfaction. This paper presents an implementation of a system of intelligent products, developed under the scope of the GRACE project, where an agent-based solution was deployed in a factory plant producing laundry washing machines. The achieved results show an increase of the production and energy efficiency, an increase of the product quality and customization, as well as a reduction of the scrap costs.
\end{abstract}

(c) 2015 Elsevier Ltd. All rights reserved.

\section{Introduction}

Traditionally, manufacturing domain operates in a conservative market place, with plants running for more than 10 years, unanticipated downtime provoking significant losses and using Enterprise Resource Planning (ERP) systems focused on mass production. However, worldwide markets are imposing strong requirements in terms of cost, quality, customization and responsiveness (ElMaraghy, 2006). These requirements create new needs related to the introduction of new manufacturing paradigms and methods, and especially decentralized approaches, being manufacturing companies forced to adopt more modular, flexible, adaptive and reconfigurable systems aiming to remain competitive in this severe context. Particularly, manufacturing systems should cope with the high degree of complexity required to implement agility, flexibility and reactivity in customized manufacturing (Morel, Valckenaers, Faure, Pereira, \& Diedrich, 2007).

The factory of the future can be seen as a large and complex system of systems, where collaboration takes place to reach global goals, complemented with other key issues like intelligence, responsiveness and adaptation. The achievement of the factory of the future raises several challenges, namely interoperability,

\footnotetext{
*Corresponding author at: Polytechnic Institute of Bragança, Campus Sta Apolónia, Apartado 1134, 5301-857 Bragança, Portugal.

Tel.: + 351273303065 ; fax: + 351273313051.

E-mail addresses: pleitao@ipb.pt (P. Leitão), nrodrigues@ipb.pt (N. Rodrigues), jbarbosa@ipb.pt (J. Barbosa), Claudio_Turrin@whirlpool.com (C. Turrin), Arnaldo_Pagani@whirlpool.com (A. Pagani).
}

plug and play, self-adaptation, reliability, energy awareness, cross layer integration, event propagation and management. The achievement of these advanced functionalities requires the use of new paradigms, such as Cyber-Physical Systems (CPS) (Rajkumar, Lee, Sha, \& Sankovic, 2010; Colombo et al., 2014). In opposite to the concept of Internet of Things (Gershenfeld, Krikorian, \& Cohen, 2004), where the focus is more in the interconnection of cooperative objects, the CPS concept also considers the computational decision-making components to provide intelligence, responsiveness and adaptation. In fact, CPS combines mechatronics and Information Technology (IT) to control physical processes and systems, designed as a network of interacting software and hardware components, devices and systems, each one with a higher level of autonomous decision making. CPS focus intelligent, dynamic and self-* large-scale systems, such as manufacturing and processes plants, electrical power grids and pipelines, logistics and transportation.

Industrie 4.0 is a German initiative constituting a concretization of CPS to promote the computerization of traditional industries aiming to achieve intelligent factories characterized by adaptability, efficiency, reliability, safety, and usability, and supporting the integration of the supply chain (Böhler, 2012). This vision, seen as the 4th industrial revolution, considers distributed intelligence and self-* methods, e.g., adaptation, configuration and diagnosis. In the United States, a similar concept is the Industrial Internet that aims the integration of complex physical machinery with networked sensors and software. The Industrial Internet Consortium comprise General Electric, AT\&T, Cisco Systems, Intel, IBM and United States government and has a broader scope than 
industrial production, namely covering also smart electrical grids, smart transportation and smart healthcare.

The concept of intelligent product (Meyer, Främling, \& Holmströmc, 2009; McFarlane, Giannikas, Wong, \& Harrison, 2013) is a new industrial manufacturing control paradigm, aligned with CPS. Intelligent products carry the knowledge about their characteristics, wirelessly connected to share, in real-time, information about their state or environment, or to communicate with other cooperative objects of the Internet of Things (IoT). Intelligent products collect and store data to support the implementation of monitoring, traceability and decision-making functions.

The use of intelligent products can bring important benefits in the Industrie 4.0 and Industrial Internet contexts, namely:

- Establishment of a product-driven production approach (i.e. the product takes the initiative during the plan execution (Wang, Huang, \& Dismukes, 2004)).

- Improvement of the entire life-cycle of the product, comprising the design, production, distribution, operation and end of life (Parlikad \& McFarlane, 2007) phases.

- Improvement of the product quality and performance through the application of self-* methods, such as self-learning, selfdiagnosis, self-adaptation and self-optimization.

- Improvement of the next generation of the product.

Multi-agent systems constitute a software engineering paradigm, derived from distributed artificial intelligence, which is based on a set of distributed, autonomous and cooperative entities, known as agents (Ferber, 1999; Wooldridge, 2002; Leitão, Mařík, \& Vrba, 2013). Each agent possesses its own knowledge and skills, being the intelligent global system behaviour emerged from the interaction among the distributed agents. Following the principle of "divide to conquer", multi-agent systems replace the centralized control by a decentralized functioning, allowing reaching a high degree of flexibility, robustness and responsiveness, which are not provided by centralized solutions. Multi-agent systems approach is being applied in several domains, namely electronic commerce, manufacturing and logistics (see e.g., Leitão (2009) for a deeper analysis), and constitutes a suitable technology to implement the intelligent product paradigm.

The objective of this paper is to explore the industrial control paradigm of product intelligence by describing a practical implementation in the manufacturing domain based on the experience gathered during the execution of the GRACE (Integration of process and quality control using multi-agent technology) project (http://grace-project.org/). During this project, a product-driven agent-based solution was installed in a factory plant producing laundry washing machines aiming the integration of process and quality control. The intelligence embedded in distributed and cooperative agents, and particularly in the products, allowed to self-adapt and self-optimize the production and product parameters, improving the production and energy efficiency and the product quality, as well as reducing the costs of scraps.

The paper is organized as follows: Section 2 overviews the concepts and deployment challenges regarding the product intelligence concept and Section 3 overviews the main principles of the GRACE multi-agent system to integrate the process and quality control. Section 4 introduces the concept of intelligent product deployed in an industrial factory plant according to the perspective of the GRACE project, namely describing the embedded intelligence for the on-line decision-making during the production phase and analysing the achieved results. Section 5 presents the intelligence mechanisms for the operation phase to be embedded in the agents hosted in each individual washing machine. Finally, Section 6 rounds up the paper with the conclusions.

\section{Intelligent products: concepts and deployment challenges}

The concept of intelligent products is being studied by the research community in intelligent manufacturing field (a state of the art in the field can be found in (Meyer et al., 2009; McFarlane et al., 2013)).

\subsection{Concepts and theory}

The definition of intelligent product is not unique. A possible definition is provided by Meyer et al. (2009) that defines intelligent product as a "physical order or product instance that is linked to information and rules governing the way it is intended to be made, stored and transported that enables the product to support or influence these operations". This definition highlights the processes regarding to manufacturing and distribution phases, but should be extended by considering also the operation phase of the product, i.e. when the product is performing the functions for what was created and produced, supporting the closed-loop Product Lifecycle Management (PLM) systems, as described in Kiritsis (2011).

According to Wong, McFarlane, Alunad Zaharudin, and Agarwal (2002), an intelligent product possesses the following five characteristics (or partially):

a) The product possesses a unique identification.

b) The product is capable of communicating with its environment.

c) The product can store data about itself.

d) The product deploys a language to share its features, requirements and plans.

e) The product is capable of participating in relevant decisionmakings to its own destiny.

Therefore, an intelligent product comprises IT in the form of software, microprocessors and sensors, and is able to collect and process information and generate knowledge, and even provide reasoning capabilities, as illustrated in Fig. 1. The traceability and identification of products are generally processed by using biometric information or instrumenting the product with related technologies such as barcode or RFID (Radio Frequency Identifier). Each intelligent product provides a set of product related services, e.g., monitoring, data analytics, self-diagnosis and self-maintenance.

Meyer et al. (2009) introduced a three dimensional framework to analyse the intelligence product concept, based on the levels of intelligence, location of the intelligence and aggregation level of intelligence. In terms of levels of intelligence, different levels can be defined, namely:

- Passive: i.e. cooperative objects can only collect and store information.

- Active: i.e. cooperative objects can collect, store, process information (even generating new knowledge), and retrieve information.

- Intelligent: i.e. cooperative objects can reason over collected and stored data and participate in decision-making processes.

The second dimension is related to the location of the intelligence, which can be completely outside the physical product, e.g., running in a remote PC, or located at the physical product itself (in this case, the computational and storing capabilities are required and network connectivity is necessary to interact with other entities). The two possibilities to locate the intelligence are illustrated in Fig. 1. The third dimension is related to the kind of aggregation of the product intelligence, ranging from intelligence only about the product itself or also aware of the components that it is made of (Meyer et al., 2009). 


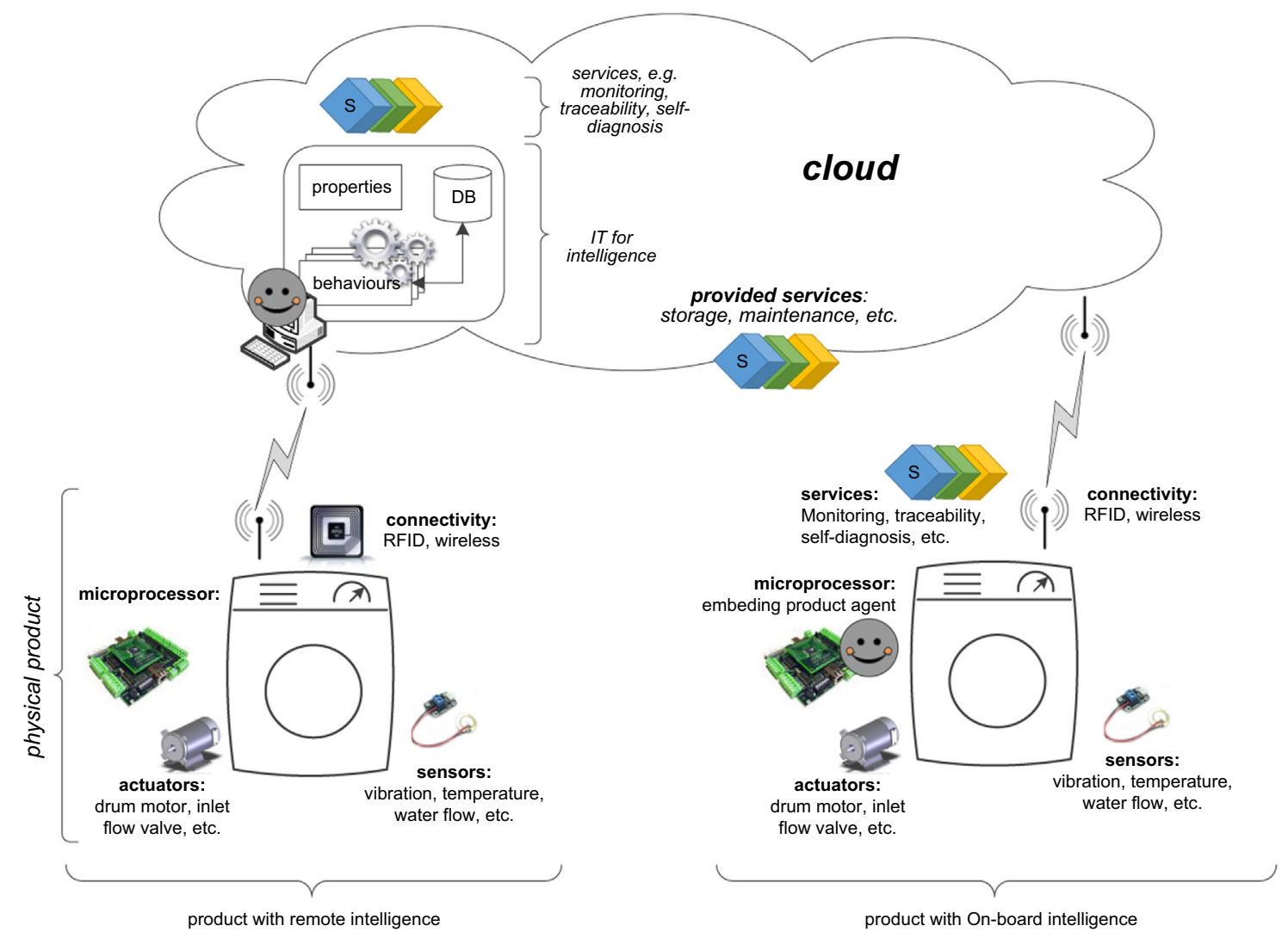

Fig. 1. Intelligent product concept.

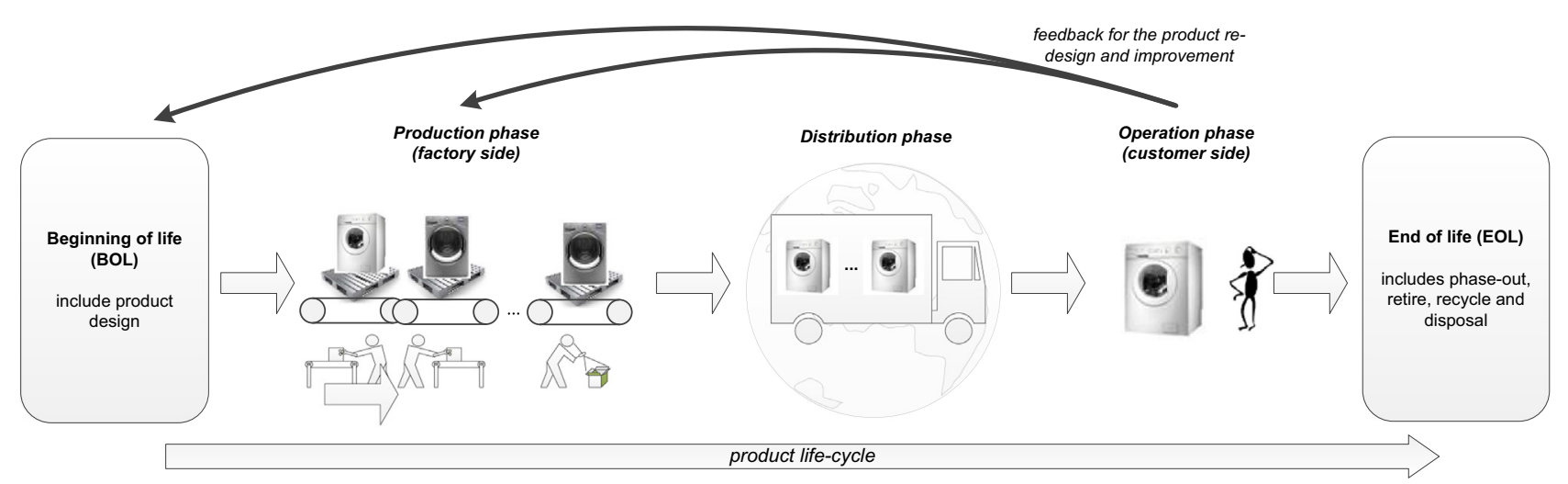

Fig. 2. Product intelligence along the product's life-cycle.

Additionally to these three dimensions, a fourth dimension focusing the product life-cycle is necessary to be considered. In fact, the roles and functionalities of intelligent products, and also the provided services, are varying along its life-cycle, e.g., considering the design, production, distribution, operation and end of life phases, as illustrated in Fig. 2.

During the production phase, the intelligent product is capable to collect data and to decide the way the product is being produced in a product-driven approach (e.g., implementing functions of monitoring, traceability, re-routing, self-adaptation and self-optimization of the production process and product configuration/customization). In the distribution phase, the intelligent product controls the safety and conditions during the transportation by registering data about e.g., transportation time, acceleration, temperature, inclination and breaks, and applying functions of monitoring and data analysis. At last, in the operation phase, the intelligent product senses the functioning conditions and controls the functioning behaviour during its operation being capable to self-adapt to condition changes (provoked by external factors, e.g., temperature, or internal factors, e.g., materials wearing). At this stage, several functions may be implemented, namely monitoring, self-diagnosis and maintenance. In the begin of life and end of life phases, similar functions are expected, e.g., aiming to improve the re-design of the product based on the feedback from posterior phases, and the way the product is recycled to improve or adapt its behaviour to condition changes.

Aiming to achieve the described features, potential functionalities that product intelligence can provide are the collection, storage and retrieval of data, and also the decision-making. These functionalities, e.g., provided by intelligent agents, can be encapsulated as services, using the service-oriented principles, and then simplifying the integration of intelligent products in bigger systems. For this purpose, a kind of middleware located in the cloud, may manage the services offered by the cooperative objects (e.g., 
other intelligent products), including data collection and storage, and decision-making functionalities.

The concept of intelligent product has several instantiations, namely the active products introduced by Sallez, Berger, and Trentesaux, 2009, which are defined as the product capability "to act based on the real state of the system", being composed of a physical product embedded with IT capabilities. Another interesting concept is the holon, introduced by Koestler (1969), which by its nature comprises an information part and a physical part (Winkler \& Mey, 1994), and particularly a product holon that may include the physical product and an intelligent agent to implement the information part. However, product holons usually found in holonic manufacturing control architectures, such as PROSA (Brussel, Wyns, Valckenaers, \& Bongaerts, 1998) and ADACOR (Leitão \& Restivo, 2006), focus the management of the product model and knowledge and not necessarily the real time management of the product life-cycle functions. Similar to the holon concept is the mechatronic unit concept (Lüder et al., 2010) that also relies on combining IT with the physical devices, but here the focus is on hardware resources and not in products being produced, transported and stored. Smart objects (López, Ranasinghe, Patkai, \& McFarlane, 2011) and physical BDI agents (Garcia Higuera \& de las Morenas, 2014) also consider the inspiration on intelligent products.

Several related concepts to intelligent products have been developed throughout the years, preparing the path and enhancing the concept of intelligent product. Auto-ID ${ }^{1}$ is one initiative that is paving the way by promoting the open-standardization, the use of RFID technology and data repository systems as the way to enable an intelligent decision making and product control of the item associated to the RFID tag (McFarlane, Sarma, Chirn, Wong, \& Ashton, 2003). Several research Auto-ID institutes are spread all over the world, each one focusing on different intelligent products topics, ranging from hardware to business process and applications, targeting several application domains such as manufacturing and supply chain.

European Projects are also steering the development of intelligent products related technology and concepts. One of the first of such projects was the EU FP6 PROMISE (Product lifecycle Management and Information tracking using Smart Embedded systems) (Anke et al., 2008) that firstly coined the term PEID (Product Embedded Information Device), which primary's goal was to design and develop a seamless information exchange architecture covering all the PLM phases, setting foundation on the use of RFID, wireless communication and innovative business models. The PEID concept enables the link between the product and its environment that, in the most simplistic way, is only responsible for its unique identification that could additionally have built-in computations capabilities. In fact, for such diversity of functions and intelligent products capabilities, the PROMISE consortium has defined 5 PEID groups, starting with identification only capabilities for type 0 and growing in terms of features and capabilities for type 4. This group division is also aligned with the levels of intelligence proposed by Meyer et al. (2009).

The ID@URI approach, i.e. a unique identification associated with a computer address, is used as the informational ground base to the development of a GUPI (Globally Unique Product Identifier). This solution is being developed at the Helsinki University of Technology under the name of DIALOG (Distributed Information Architectures for collaborative LOGistics) and has a similar approach to the one promoted under the Auto-ID initiative. One of the major premises taken by the DIALOG approach is that every physical agent, i.e. a product, must be represented by a virtual

${ }^{1}$ http://autoidlabs.org. counterpart, i.e. an agent, during it whole life-cycle, handling all the physical agent informational part such as location, service lookup or any other transactions (Kärkkäinen, Ala-Risku, \& Främling, 2003).

Although the real applications of product intelligence models are still very limited, some examples can be found in logistics, e.g., addressing the dynamic routing in the intermodal transportation problem (Giannikas \& McFarlane, 2012). The warehouse management is also addressed by using a product intelligence solution (Giannikas, Lu, McFarlane, \& Hyde, 2013), particularly focusing on defining adaptive storage location strategies and on the dynamic order-picking rescheduling. Biological inspiration was used to support the product intelligence solution that has been developed for the dynamic routing within a flexible manufacturing system (Sallez et al., 2009).

\subsection{Deployment challenges}

The deployment of product intelligence introduces several challenges that deserve to be discussed. The first critical issue is how to collect the data, and also which type of data should be handled (note that a proper selection of the type of data impacts the amount of data retrieved). Operators and automatic systems must actively acquire, clear and load data into appropriate systems and maintain the data properly. Important at this stage is the integration of RFID technology for the automatic identification of products and wireless sensor networks for a transparent collection and transmission of data. As an example, García, Chang, and Valverdeet (2006) analyse the impact of using automatic identification and data capturing technologies based on the RFID technology in highly automated distribution centres following the intelligent products principles.

The second issue is related to the storage and retrieval of data. Particularly, the use of semantics and ontologies (Gruber, 1995) to represent the acquired and exchanged knowledge, providing a common understanding, is crucial to ensure interoperability among distributed entities and legacy systems. Since, the current RFID technology, usually used to identify products, does not allow to store a big amount of information, as well the internal memory or database of intelligent entities, the need for cloud systems to store data is gaining an increasing importance. In this context, speed, security, safety and trust are important issues to be considered during the access, storage and retrieval of data, and particularly when cooperative objects may interact or use the cloud. As example of security, a car producer is interested to access the data collected by the associated intelligent products, probably stored in the cloud, for posterior implementation of maintenance or product re-design tasks, but cannot access to data of intelligent products associated to one of its competitors. On the other hand, can one car producer trust on the data that is being collected by its intelligent products? Concerning the increasing importance of cyber-security issues, it is important to consider the use of private clouds to store the collected data and to encrypt the exchanged messages.

Another issue is related to the decision-making. By nature, data is passive, being necessary to implement proper mechanisms to transform the available data into knowledge supporting decisionmaking tasks. For this purpose, intelligent products can reason about their desired characteristics, e.g., using artificial intelligence techniques or data mining techniques (see e.g., Thomas and Thomas (2013) where data mining techniques were used for product-driven systems), introducing data analytics and exploring the incorporation of self-* properties like self-diagnosis, selfadaptation and self-healing. More advanced functionalities can be designed, e.g., sending notifications for the product producers 
asking maintenance interventions or support from the other cooperative objects.

The integration of the human-in-the-loop and legacy systems are also very important topics, being required the use of standard interfaces. The humans are important pieces in these systems, with human-centred technology playing a special role (Boy, 2012). The success of new manufacturing paradigms requires their inclusion in the automation loop, avoiding the errors exhibited of some past paradigms, e.g., CIM (Computer Integrated Manufacturing). The interaction with humans can be achieved by the use of mobile devices, such as the smartphones and tablets, which can act as the interface for a bidirectional communication. First, the intelligent product can act in an active way and inform the user of overpassed functioning limits or just issuing daily (or periodically) working reports. On the other direction, the user can enquire its product for the current status diagnosis, which will start an internal product check-up, just to give one example. Regarding the industrial automation, the use of standard interfaces, e.g., OPCUA (Open Process Control - Unified Architecture) and DPWS (Device Profile for Web Services), should be followed for the transparent interconnection with hardware automation systems.

The product complexity may affect the application of the product intelligence concept. In fact, this concept can be applied to distinct application areas, such as electrical grids, traffic management, building automation, logistics, transportation and manufacturing. However, in some situations, the identification of the products is difficult, e.g., the product in smart grids, i.e. energy, is hard to sense, being necessary to discretise this kind of products.

The deployment of product intelligence in several situations, and particularly when the storage and intelligence is embodied in the physical product, e.g., clothes, requires the miniaturization of electronic devices, namely microchips and sensors using nanotechnology. In spite of the long road to be covered in the near future, the concept of communicating material (Jover, Thomas, Leban, \& Canet, 2010) is related to the use of a plethora of elements, with storage and communication capabilities, that are embedded in the products, e.g., clothes and wood materials. Also important are the use of batteries to provide autonomy to these intelligent devices and the hostage of intelligence (e.g., using agent technology) in low-cost devices, such as Raspberry Pi or Arduino controllers.

\section{GRACE Multi-agent system for integrating process and quality control}

The objective of the GRACE project is to develop a system that integrates process and quality control for the implementation of self-adaptation and self-optimization procedures leading to the adjustment of variables of production process and product configuration. The need to develop such system comes from the idea to achieve a more efficient and adaptive production line sustained by real-time feedback control loops that uses the quality control information to adapt the process and product parameters. Nowadays, this feedback control loop is not properly handled in realtime, culminating in a non-optimal process adaptation and product customization, reflected in terms of non-optimal energy and production efficiency and product quality.

For this purpose, multi-agent systems principles are applied taking advantage of their intrinsic characteristics. A crucial assumption considered in this work is to keep the existing lowlevel control based on a network of Programmable Logic Controllers (PLCs) running state-of-the-art IEC 61131-3 control programs. These PLC programs ensure the real-time responsiveness and the use of the multi-agent system, located at higher control level, provides an infrastructure for the integration of process and quality control by means of intelligence and adaptation functionalities (Rodrigues et al., 2013b).

The GRACE multi-agent system approach introduces several differences when comparing with existing holonic and agentbased approaches, such as PROSA and ADACOR. In fact, PROSA and ADACOR are generic holonic architectures and GRACE focuses on the product-driven production where the intelligent products play a central role. Additionally, GRACE aims the adaptation of the process and product variables to achieve the improvement of the product efficiency and quality, and not particularly the reconfiguration of the production process towards an agile manufacturing system.

\subsection{GRACE Multi-agent system}

The multi-agent system application distributes the manufacturing functions by a society of agents representing the manufacturing objects. In particular, several types of agents were considered, namely the Product Type Agent (PTA), Product Agent (PA), Resource Agent (RA) and Independent Meta Agent (IMA) (Rodrigues et al., 2013b). PTAs represent the catalogue of products that can be produced by the production line and contain the process and product knowledge required to produce the product. PAs manage the production of product instances in the production line (e.g., washing machines and drums). Note that products might not only be final products commercialized by the OEM (Original Equipment Manufacturer) company but also intermediate parts/ components.

RAs are associated to the physical resources placed in the production line, such as robots, quality control stations and operators. Specializations of the RA were developed focusing particular specifications while inherit the RA functionalities, namely Machine Agents (MA) representing the mechatronic equipment, such as robots and machines, and Quality Control Agents (QCA) associated to the quality control stations.

IMAs introduce a kind of hierarchy in the decentralized system allowing to implement global monitoring and optimized global operating strategies for the system. These entities are continuously collecting information of the production system from RAs and PAs, and performing data analytics to detect deviations or trends on the system operation that will require the optimization of the operating parameters. Based in their broader perspective, they send these optimized operation parameters as guidelines to the other agents, namely RAs and PAs.

The manufacturing components must cooperate to produce a product, and must coordinate their actions according to the product dependencies and production plan, as illustrated in Fig. 3.

Particularly, the integration of process and quality control aiming the implementation of feedback control loops for the self-adaptation of process and product configuration is achieved by the interaction among these types of agents.

\subsection{Product agent functionalities}

As on-demand production is typically a product-driven approach, PAs assume critical importance in the GRACE multiagent system architecture. Mainly, product agents contain the knowledge, intelligence and capability to adapt themselves autonomously and are associated to physical mechatronic products being produced. The PA together with the mechanical, electronic and software based components of the physical product, forms the intelligent product. The PA concentrates its knowledge mainly about the product it represents, being the knowledge and intelligence about the sub-products that the product is made of associated to the PTA agent. 


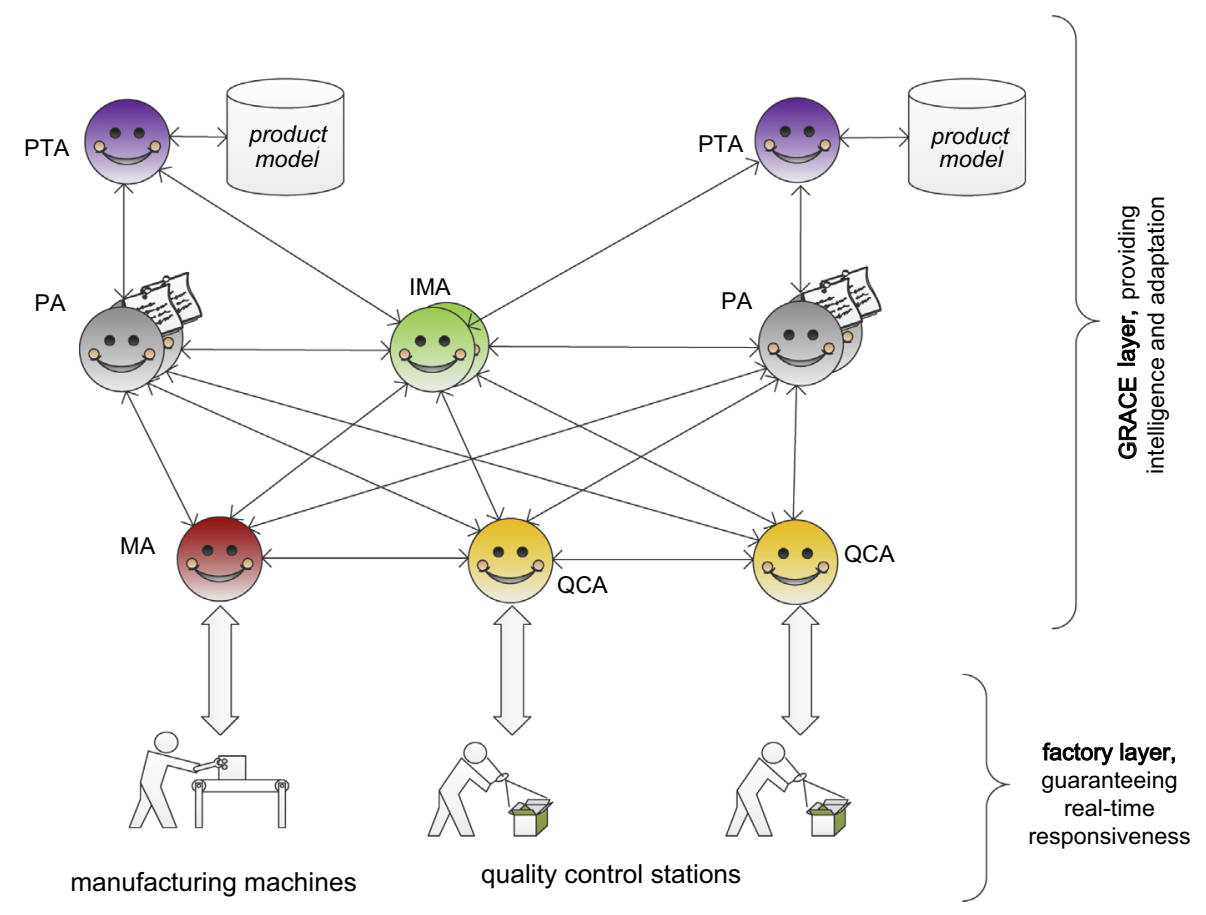

Fig. 3. GRACE multi-agent system architecture.

PAs possess the process plan to produce the product and are responsible for the following main functions (provided as intelligent services) along their life-cycle, during the production phase:

- Management of the production process of the product by interacting with RAs to coordinate their actions according to the production dependencies and the production plan.

- Data collection along the production line about the production execution of the product aiming to support the monitoring, traceability and data analysis.

- Re-routing of pallets and particularly adaptation of the control structure to face the current situation of the production process.

- Optimization/adaptation of the processing and inspection operations by correlating the collected processing and inspection data.

- Customization of the product by considering the adjustment of the parameters used by the on-board controller that regulates the product operation.

PAs, by interacting with RAs, are continuously acquiring the information related to the production process, namely the performance indexes, $P_{j}$, of the processes executed at the workstations, which reflects the degree of success of the execution of such processing or inspection operation. The $P_{j}$ parameter is expressed in the interval $[0,1]$, being 1 the representation of a good process and 0 a bad process. The determination on this parameter, performed at the end of each processing or inspection operation by the associated RA, is dependent of the particularities of each process; an example, the MA associated to the bearing insertion station collects data correlating the torque and depth variables during the execution of the operation, and at the end it determines the $P_{j}$ by calculating the proximity of some pre-defined points of the achieved curve with the optimal curve for this process.

GRACE also introduces the Material/Process/Function/Quality (MPFQ) model (Rodrigues et al., 2013a) that correlates materials (something that is needed to produce a product), processes (operations to process and transform materials into final goods) and also functions (characteristics of a product item) to the quality of the product being produced. In this work, the quality is expressed in several quality indexes $\left(Q_{i}\right)$, particularly those related to noise, energy saving, component conformity, assembly conformity, no-leakage, washing performance, safety and green footprint. Considering only the contribution of the processes, the quality index of a product is given by:

$Q_{i}=\sum_{j=1}^{n} P_{j} \times S Q_{i j}$

where $n$ is the number of workstations along the production line, $P_{j}$ is the performance index of the process performed at the workstation $j$ and $S Q_{i j}$ is the parameter correlating the contribution of each process to the final quality of the product.

The use of the feedback information related to the results of processing/inspection operations and the MPFQ concept can be used to design proper self-adaptation mechanisms according to the particularities of the production system. Examples of these mechanisms, which will be detailed later, are the configuration of the inspection tests to be executed by the quality control stations, through the selection of the proper algorithms and/or testing parameters, and the customization of the control programme that regulates the product operation.

PAs can run on the cloud, particularly using High Processing Computing (HPC) environments to achieve faster processing capabilities, and can also dynamically self-organize in different clusters.

\section{Embedded intelligence for the on-line decision-making in the production phase}

This section details the use of product intelligence to achieve self-adaptation and self-optimization in the production phase of the life-cycle, namely by instantiating the GRACE architecture to be applied in an industrial factory plant. For this purpose, several self-adaptation mechanisms were implemented, namely the monitoring of the quality achievement, the adjustment of the functional tests plan and the customization of the parameters of the appliance's on-board controller. 


\subsection{Industrial use case}

The use case considered in this work to apply the GRACE system is an industrial production line that produces a set of different models of laundry washing machines. A simplified schema of the production line is illustrated in Fig. 4, being composed of the washing unit lines $\mathrm{A}$ and $\mathrm{B}$, and the assembly line, complemented by the drum and cabinet lines. Each one of these lines comprises a set of workstations organized in a sequential manner according to the assembly process.

The pallets carry the products to be produced through the production line using a conveyor system, according to a process plan that specifies the sequence of operations needed to produce that washing machine model. Workstations disposed along the production line perform the operations specified in the process plan, namely processing operations (e.g., seal insertion or tube welding), inspection operations (e.g., gap control or assembly visual check) and manual operations (e.g., electronics assembly). Each pallet is equipped with an active RFID tag, which has a unique identifier and is capable to store some relevant information from processes that are being executed. More detailed data related to the production execution, e.g., results and measurements of each operation performed in the production area, are dynamically and real-time stored in a central SQL server database.

The designed agent-based system was implemented using the JADE framework (Bellifemine, Caire, \& Greenwood, 2007), and deployed in the factory plant by installing the agents in different PCs distributed along the production line. Each PA is running in a remote $\mathrm{PC}$ that is connected to the physical product through a TCP/ IP network by using the RFID tag placed in the product pallet: the RFID readers placed in the workstations allow to identify the current location of the product.

According to the framework established by Meyer et al. (2009), the positioning of the GRACE PA agent as intelligent product is illustrated in Fig. 5.

The options concerning the location, level and aggregation of the intelligence were performed taking into consideration the end user requirements and the factory constraints. In terms of the level of intelligence, PAs should be able to reason and apply selfadaptation mechanisms using the gathered production data, while in terms of the aggregation of intelligence, the objective was to consider intelligence about the product itself, namely its quality. Finally, in terms of location of the intelligence, a remote approach was considered due to the factory constraints and the need to preserve the current low-level control running in the production line.

\subsection{Monitoring the quality achievement}

PAs are performing an online monitoring of the evolution of the product quality along the production process by analysing the evolution of the quality indexes. This mechanism permits to detect, in real time and at any point of the production line, generating a yellow warning when the desired quality of a washing machine being produced is at risk or a red warning when it is not possible anymore to achieve the desired quality, even if all the remaining operations would be performed with a performance of $100 \%$. In this last case, the agent may decide to stop the production of the washing machine, removing it from the production line. The expression to trigger a red warning for the quality index $i$ at station $k$, where $k \leq n$, is the following:

$\sum_{j=1}^{k}\left(1-P_{j}\right) \times S Q_{i j} \geq \operatorname{gap}_{i}$

where $\operatorname{gap}_{i}$ is the maximum allowed margin for missing quality for the index $i$.

Fig. 6 illustrates the evolution of the quality index related to the noise quality parameter along the production stage for two different washing machines (id "11321003670" and id "11321003673") and considering an admissible 10\% for the maximum gap for the desired final quality. As shown, the washing machine id "11321003670" was produced fulfilling the desired quality (at the end, the gap for the optimal quality is $8 \%$ which is within the accepted margin for the product quality). However, the washing machine id "11321003673" has a red signal at the execution of the operation $\# 9$, which means that the PA decided, at that point, to remove the washing machine from the production line since the desired quality will never be reached even if all the remaining processes are be executed with a success of $100 \%$.

The application of mechanisms for monitoring the quality achievement can lead to an increase of the production efficiency and a reduction of the scraps from $3 \%$ to $5 \%$. These estimations are supported by the capability to identify earlier washing machines being produced that will never reach the desired quality due to quality unconformities and remove them from the production line (i.e. saving time and money since good parts are not assembled in non-acceptable washing machines).

\subsection{Adaptation of the functional tests plan}

A similar procedure is used to adjust the set of functional tests to be performed in the Functional Test Area, located near the end

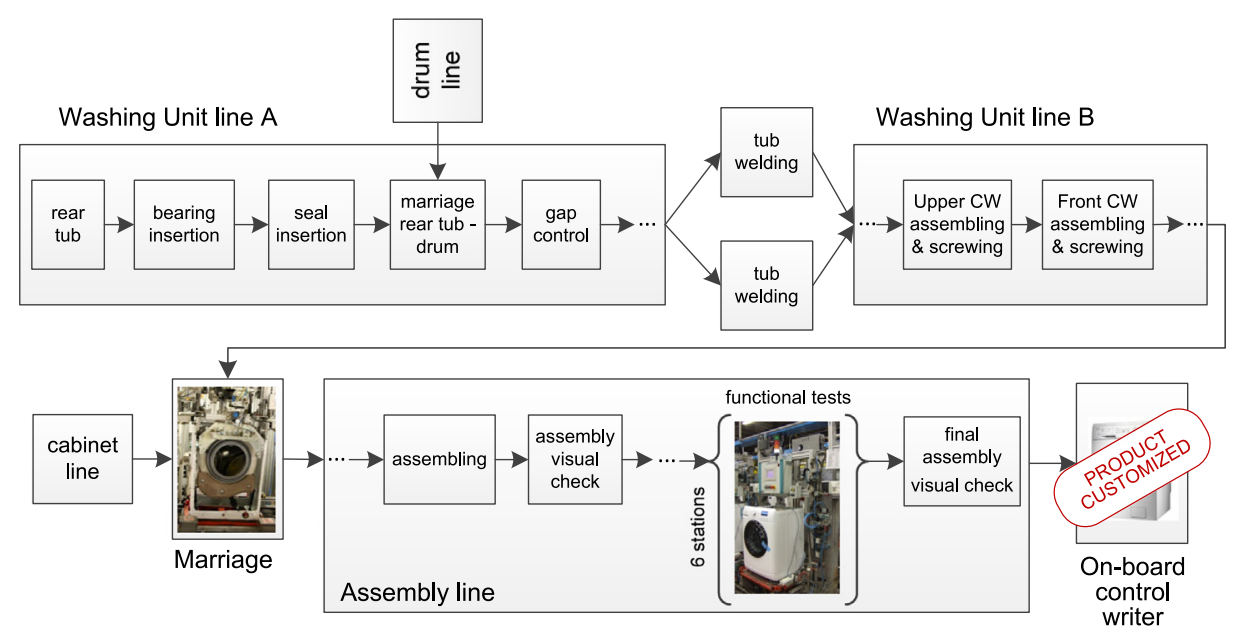

Fig. 4. Layout of the use case production line. 


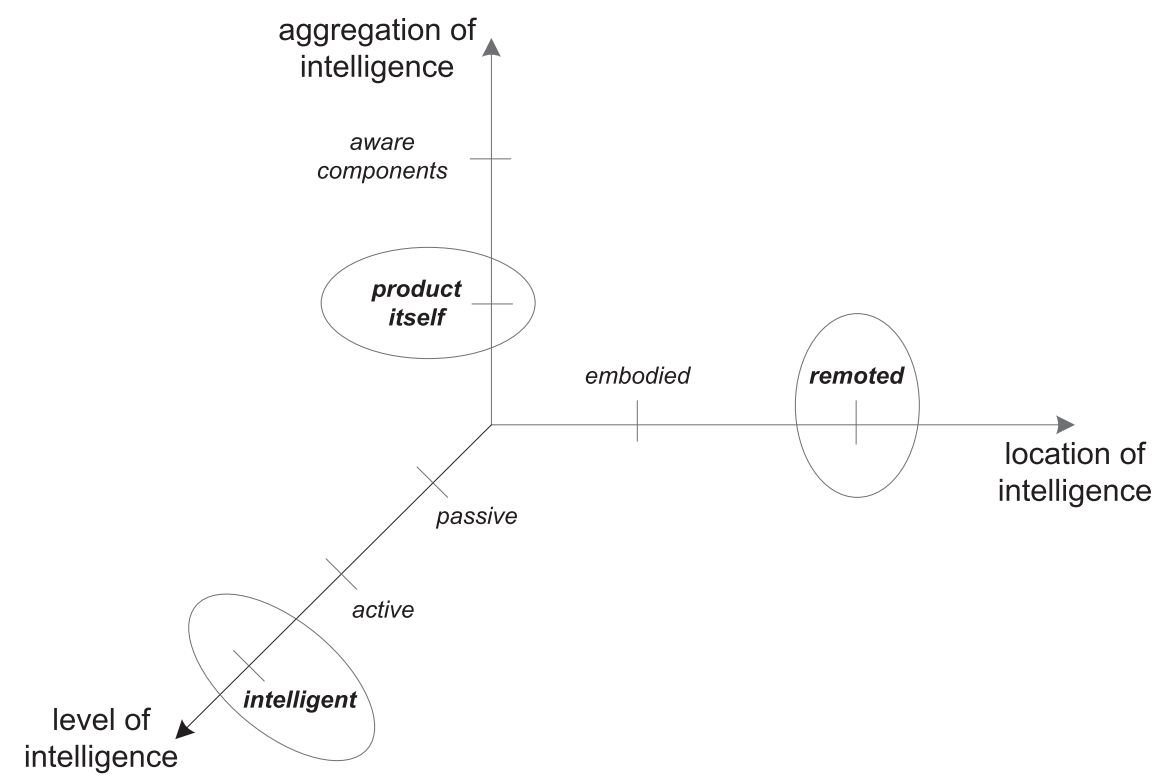

Fig. 5. Positioning of the GRACE Product Agent as Intelligent Product according to the framework established by Meyer et al. (2009).

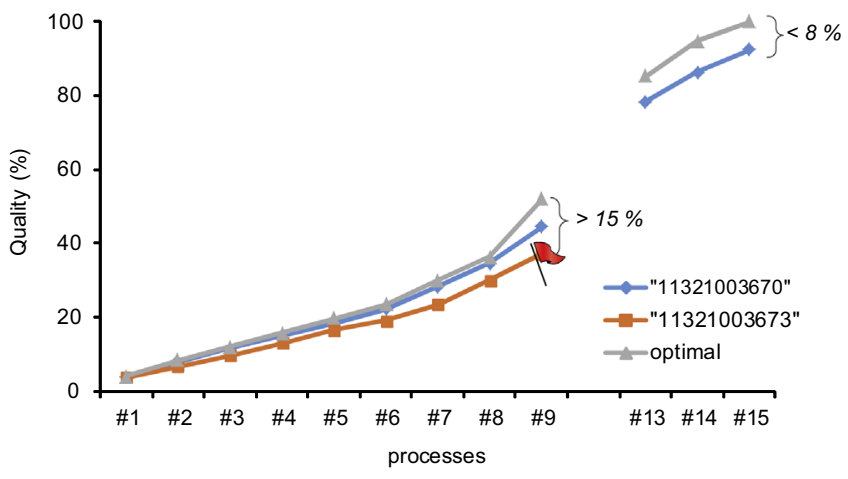

Fig. 6. Evolution of the products noise quality index along the production line.

of the production line, which was designed to verify that the product components are properly assembled.

The execution of the functional tests uses a parameterized testing rule that is composed by a list of "elementary action blocks", which sequence follows Boolean rules conditioned by events like the success or the failure of one test/action contained in a block. Acting on the variable conditions, it is possible to change the sequence of the testing plan (i.e. if a value is inside or outside a boundary, the test can jump on a sequence of blocks instead of others). PAs can customize the default testing plan by:

- Adjusting the sequence of the tests for a specific washing machine, e.g., removing some unnecessary tests or adding new ones.

- Personalizing the operator messages, e.g., highlighting particular aspects that deserve a more detailed test.

The function embedded in the PA considers the gathered information along the production line for each process (i.e. the $P_{j}$ information), and the advices provided by the IMA, to calculate the set of variables that define the sequence of tests in the generic testing rule that is used by the PLC to control the functional test station. For this purpose, the set of collected $P_{j}$ is correlated with the MPFQ table (representing the dependencies between the causes, i.e. $P_{j}$, and the consequences, i.e. the $v r_{k}$ parameters), taking into consideration the model of the washing machine, as illustrated in Fig. 7.

As an example, the calculation of $\operatorname{var}_{1}$ (related to the noise index) considers the $P_{j}$ collected along the production line to customize the testing rule, and particularly those related to the bearing insertion and vibration analysis stations since they are the main causes for this performance deviation.

The calculated var $_{k}$ parameters are written in the "Table of Parameters", which are posteriorly used by the testing rule running in the PLC. When matching the generic testing rule and the $v r_{k}$ parameters written in the "Table of Parameters", the testing plan is customized for the particularities of the produced washing machine, performing measures and sequences adapted for the specific appliance under test. As example Rodrigues et al. (2013a):

- If the $v a r_{1}$ is set in the interval $[0.7,1]$ : no additional message is displayed to the operator.

- If the $v r_{1}$ is set in the interval [0.3, 0.7]: a message is displayed to the operator, warning to take special attention during the execution of the functional test.

- If the $v{ }_{1}$ is set in the interval [0,0.3]: a different message is displayed to the operator, warning that something suspicious happened during the production that requires the implementation of additional procedures during the execution of the functional test.

The adaptation of the functional tests for each washing machine allowed to reduce, by approximately 20\%, the time devoted to perform the functional tests, which implies an increase of the production efficiency. Additionally, the introduction of more effective and self-adapted quality control procedures allows an estimated increase of the product quality and to reduce the nonconformities of about $1.5 \%$.

\subsection{Adaptation of the on-board controller parameters}

Another important and innovative control loop integrating process and quality control is related to the customization of the parameters of the on-board controller installed in the produced washing machine (and not derived from a default programme and parameters). The adaptive adjustment of the parameters of the on- 


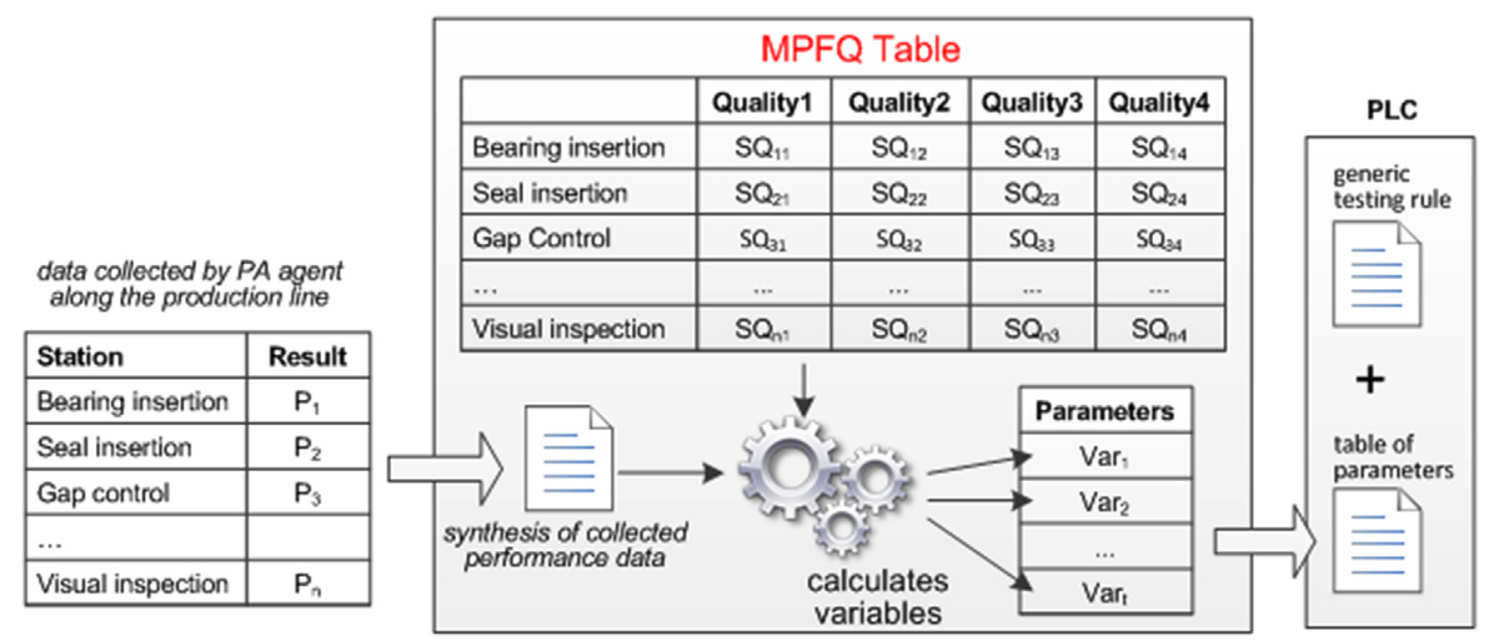

Fig. 7. Customization of the testing plan Rodrigues et al. (2013a).

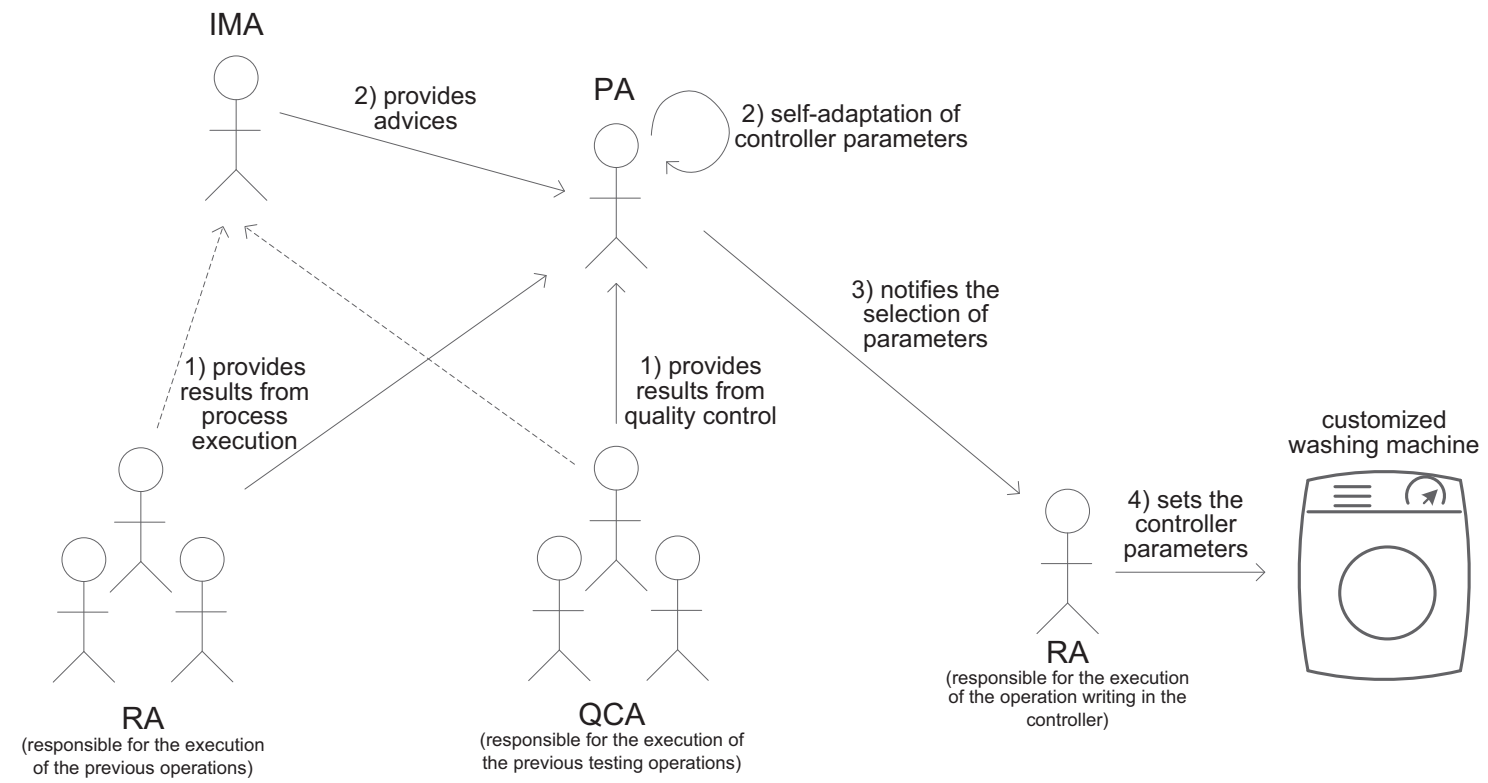

Fig. 8. Adjusting the parameters of the on-board controller of the washing machine.

board controller allows to reduce significantly the dispersion of performance between products, taking into account the results of the inspection tests performed along the production line and to keep its performance within specifications even in case of assembly problems. In this way, each final product will be calibrated for its specific characteristics in order to reach maximum efficiency and optimize compliance to specifications.

As illustrated in Fig. 8, the PA correlates the acquired knowledge along the production line using proper algorithms, also combined with the advices provided by the IMA agent, to determine the optimized parameters to be written in the EEPROM (Electrically-Erasable Programmable Read-Only Memory) of the on-board controller. This allows to adapt the functioning of the controller according to the individual characteristics of each machine, keeping its performance within the specifications. The operation of writing these parameters to the EEPROM, updating the default parameters already stored in the controller, is accomplished by a writer machine represented by a RA, which receives the optimized parameters from the PA.

The installed demonstrator considered the individual setting of the control board parameter for the inlet flow valve. This parameter is used in the calculation of the time interval the valve is open, and is therefore crucial for controlling the water consumption of the washing machine. Currently, the same value is used for all produced machines, although it is known that the valves' characteristics are different.

The adaptation of the parameters of the on-board microcontroller embedded in each washing machine allows to customize each one to achieve an optimized functioning operation and providing a significant impact on the sustainability of the washing machine operation. In particular, the calibration of the inlet flow valve permits to reduce by approximately 50\% the standard deviation in the washing machine water consumption. This reduction implies that more efficient washing machine programs can be implemented with a $5 \%$ reduction in water and energy consumption.

\section{Embedded intelligence for the operation phase}

An extension to the GRACE project is the consideration of product intelligence to cover the post-production life-cycle phases, namely the distribution and operation phases. 
For this purpose, intelligent software agents, i.e. the product agents, are not anymore running on remote PCs but instead embedded in the on-board controller of each washing machine (process performed at the end of the production line and using the on-board controller already installed in each washing machine). In particular, the PA is embedded in the mechatronic component comprising the product itself, a set of sensors and actuators and the product intelligence.

At this stage, the PA is collecting data about the functioning operation of the washing machine by using a network of sensors to measure a set of different parameters, namely the motor current value, vibration, temperature, tilt, humidity and water flow. The data gathered during the washing machine operation allows to perform on-line monitoring and data analysis, being able to selfadapt the functioning parameters written in the on-board controller (based on pre-defined correlation functions). Additionally, and besides to adjust the controller parameters, the PA is able to use data analytics to ask maintenance interventions and also provide feedback information to the owner company regarding the post-sales services and the re-design of future models. For this purpose, the huge amount of data collected during the product's functioning, stored in cloud systems, may require the application of big data techniques, probably also running in the cloud.

The main benefits of the product intelligence at this phase rely on two different vectors:

- Improvement of the quality and behaviour of the washing machine aiming the customer satisfaction.

- Improvement of the design of future models of the product to be delivered by the manufacturing company.

These vectors are linked to the levels of granularity associated to the product scope, being the first one related to the short operational functioning and customer satisfaction, and the second one related to longer feedback loops associated to strategic and redesign of future product models.

\section{Conclusions}

Product intelligence is a new industrial manufacturing control paradigm aligned with the context of cyber-physical systems, providing benefits in terms of production efficiency, energy efficiency, product quality, and product customization by offering a new kind of services, e.g., on-line monitoring, traceability, selfdiagnosis, self-maintenance and remote maintenance, aiming the customer satisfaction.

The paper explores the product intelligent concept by describing the GRACE experience acquired during the implementation of an agent-based solution in a factory plant producing laundry washing machines for integrating process and quality control.

The PA established in the GRACE architecture addresses the requirements defined by Wong et al. in the following manner:

a) It possesses a unique identification, namely using a RFID tag identifier attached to each pallet carrying on the washing machine.

b) It is capable of effectively communicating within its environment, particularly with RAs representing processing and inspection stations for allocating and adapting the execution of operations and with IMAs to get optimized advices over the production policies.

c) It stores data about itself, namely the data collected locally by the agents in charge of the process and quality control, namely MAs and QCAs, during the execution of the product along the production line. d) It displays its features or production requirements to the other system actors by using the FIPA ACL (Agent Communication Language) and the proper FIPA interaction protocols.

e) It participates in relevant decision-making processes to its own destiny, particularly defining and adapting the best parameters for the execution of processing and inspection operations, selecting the functional tests to be performed on the product and customizing the parameters to be written on the on-board controller.

The preliminary achieved results for the production phase showed an increase of the production and energy efficiency, an increase of the product quality and customization, as well as a reduction of the scrap costs. Usually, the product intelligence focuses the re-scheduling in shop-floor or supply chain. However, in this work, the innovation relies on the use of product intelligence to provide self-adaptation and self-optimization of process and product configuration.

The deployment of such hybrid intelligent product solution, and particularly the intelligence levels, can be performed incrementally and in a modular manner according to the different phases of the product's life-cycle. In fact, at the moment, the production phase was deployed and tested in the Whirlpool factory plant, being the future work related to testing the product intelligence embedded in the washing machines for the operation phase, since it requires longer feedback loops.

\section{Acknowledgements}

This work has been partly financed by the EU Commission, within the research contract GRACE coordinated by University Politecnica delle Marche and having partners SINTEF, AEA srl, Instituto Politécnico de Bragança, Whirlpool Europe srl, Siemens AG.

\section{References}

Anke, J., Wolf, B., Hackenbroich, G., Do, H., Neugebauer, M., \& Klein, A. (2008) PROMISE: product lifecycle management and information tracking using smart embedded systems. In: M. Mühlhäuser, \& I. Gurevych (Eds.), Handbook of research on ubiquitous computing technology for real time enterprises (pp. 559566). Hershey, PA: Information Science Reference. http://dx.doi.org/10.4018/ 978-1-59904-832-1.ch025.

Bellifemine, F., Caire, G., \& Greenwood, D. (2007). Developing multi-agent systems with JADE. Wese Sussex, England: Wiley.

Böhler, T.. "Industrie 4.0 - Smarte Produkte und Fabriken revolutionieren die Industrie", Produktion Magazin, 10 Mai 2012. Aaccessed on 3.4.14

Colombo, A. W. Bangemann, T. Karnouskos, S., Delsing. J., Stluka, P., Harrison, R, Jammes, F., \& Lastra, J. M. (Eds.). (2014). "Industrial cloud-based cyber-physical systems: the IMC-AESOP approach". Switzerland: Springer ISBN: 978-3-31905623-4.

Boy, G. (2012). Orchestrating human-centered design. London: Springer.

Brussel, H. V., Wyns, J., Valckenaers, P., \& Bongaerts, L. (1998). Reference architecture for holonic manufacturing systems: PROSA. Computers in Industry, 37(3) 255-274.

ElMaraghy, H. (2006). Flexible and reconfigurable manufacturing systems paradigms. International Journal of Flexible Manufacturing Systems, 17, 261-271.

Ferber, J. (1999). Multi-agent systems, an introduction to distributed artificial intelligence. Boston, USA: Addison-Wesley.

García, A., Chang, Y. S., \& Valverdeet, R. (2006). Impact of new identification and tracking technologies on a distribution center. Computers and Industrial Engineering, 51, 542-552.

Garcia Higuera, A., \& de las Morenas, J. (2014). Application of the classical levels of intelligence to structuring the control system in an automated distribution centre. Journal of Intelligent Manufacturing, 25(5), 1197-1206.

Gershenfeld, N., Krikorian, R., \& Cohen, D. (2004). The internet of things. Scientific American, 291(4), 76-81.

Giannikas, V., McFarlane, D. 2012. Product intelligence in intermodal transportation: the dynamic routing problem. In Proceedings of the 3 rd international conference on dynamics in logistics (LDIC'12), Bremen, Germany.

Giannikas, V., Lu, W., McFarlane, D. \& Hyde, J. 2013. Product intelligence in warehouse management: a case study. In V. Marik, J. L. M. Lastra, and P. Skobelev (Eds.), Proceedings of the 6th international conference on industrial 
applications of holonic and multi-agent systems (HoloMAS'13), lecture notes in computer science (pp. 224-235). Vol. 8062. Springer Berlin Heidelberg.

Gruber, T. (1995). Toward principles for the design of ontologies used for knowledge sharing. International Journal of Human and Computer Studies, 43(5-6) 907-928.

Jover, J., Thomas, A., Leban, J., \& Canet, D. 2010. Pertinence of new communicating material paradigm: a first step towards wood mass marking. In Proceedings of the new achievements in materials and environmental sciences. Nancy, France.

Kärkkäinen, M., Ala-Risku, T., \& Främling, K. (2003). The product centric approach: solution to supply network information management problems? Computers in Industry, 52, 147-159.

Kiritsis, D. (2011). Closed-loop PLM for intelligent products in the era of the internet of things. Computer-Aided Design, 43(5), 479-501.

Koestler, A. (1969). The ghost in the machine. London: Arkana Books.

Leitão, P. (2009). Agent-based distributed manufacturing control: a state-of-the-art survey. Engineering Applications of Artificial Intelligence, 22(7), 979-991.

Leitão, P., \& Restivo, F. (2006). ADACOR: a holonic architecture for agile and adaptive manufacturing control. Computers in Industry, 57(2), 121-130.

Leitão, P., Mařík, V., \& Vrba, P. (2013). Past, present, and future of industrial agent applications. IEEE Transactions on Industrial Informatics, 9(4), 2360-2372.

López, T., Ranasinghe, D., Patkai, B., \& McFarlane, D. (2011). Taxonomy, technology and applications of smart objects. Information Systems Frontiers, 13(2), 281-300.

Lüder, A., Hundt, L., Foehr, M., Holm, T., Wagner, T., Zaddach, J.-J. 2010. Manufacturing system engineering with mechatronical units. In Proceedings of the IEEE conference on emerging technologies \& factory automation (ETFA'10) (pp. 1-8).

McFarlane, D., Sarma, S., Chirn, J. L., Wong, C. Y., \& Ashton, K. (2003). Auto ID systems and intelligent manufacturing control. Engineering Applications of Artificial Intelligence, 16(4), 365-376.

McFarlane, D., Giannikas, V., Wong, A. C. Y., \& Harrison, M. (2013). Product intelligence in industrial control: theory and practice. Annual Reviews in Control, 37(1), 69-88.

Meyer, G., Främling, K., \& Holmströmc, J. (2009). Intelligent products: a survey Computers in Industry, 60, 137-148.
Morel, G., Valckenaers, P., Faure, J.-M., Pereira, C. E., \& Diedrich, C. (2007), Manufacturing plant control challenges and issues. Control Engineering Practice, 15(11), 1321-1331.

Parlikad, A. K., \& McFarlane, D. (2007). RFID-based product information in end-oflife decision making. Control Engineering Practice, 15(11), 1348-1363.

Rajkumar, R., Lee, I., Sha, L., Sankovic, J. 2010. Cyber-physical systems: the next computing revolution. In Proceedings of the 47th ACM/IEEE design automation conference (DAC'10), 13-18 June 2010( pp. 731-736).

Rodrigues, N., Leitão, P., Foehr, M., Turrin, C., Pagani, A., Decesari, R.2013a. Adaptation of functional inspection test plan in a production line using a multi-agent system. In Proceedings of the IEEE international symposium on industrial electronics (ISIE'13), May 28-31, Taipei, Taiwan.

Rodrigues, N., Pereira, A., \& Leitão, P. (2013b). Adaptive multi-agent system for a washing machine production line Lecture Notes in Computer Science. In: V. Marik, J. Lastra, \& P. Skobelev (Eds.), Industrial applications of holonic and multi-agent systems for manufacturing, Vol. 8062 (pp. 212-223). Berlin/Heidelberg: Springer.

Sallez, Y., Berger, T., \& Trentesaux, D. (2009). A stigmergic approach for dynamic routing of active products in FMS. Computers in Industry, 60, 204-216.

Thomas, P., \& Thomas, A. (2013). An approach to data mining for product-driven systems Studies in Computational Intelligence. In: T. Borangiu (Ed.), Service orientation in holonic and multi agent manufacturing and robotics, Vol. 472 (pp. 181-194). Springer-Verlag.

Wang, G., Huang, S. H., \& Dismukes, J. P. (2004). Product-driven supply chain selection using integrated multi-criteria decision-making methodology. International Journal of Production Economics, 91(1), 1-15.

Winkler, M., \& Mey, M. (1994). Holonic manufacturing systems. European Production Engineering, 19(3), 10-12.

Wong, C.Y., McFarlane, D., Alunad Zaharudin, A., Agarwal, V. 2002. The intelligent product driven supply chain. In Proceedings of the ieee international conference on systems, man and cybernetics (SMC'02).

Wooldridge, M. (2002). An introduction to multi-agent systems. UK: JohnWiley \& Sons. 